REVISTA CIENCIAS BIOMÉDICAS

PRESENTACIÓN DE CASOS CLÍNICOS



\title{
ENFERMEDAD DE MENETRIER ASOCIADA A INFECCIÓN POR CITOMEGALOVIRUS
}

\author{
MENETRIER'S DISEASE ASSOCIATED WITH AN INFECTION BY \\ CYTOMEGALOVIRUS
}

\author{
De Vivero-Camacho Rodrigo (1) \\ Muñoz-Álvarez Nelson (2) \\ Redondo-Bermúdez Cesar (3) \\ Alvis-Guzmán Nelson (4) \\ Tovar-Correa Luz Esthella (5)
}

Correspondencia: stellatovar2009@hotmail.com

Recibido para evaluación: abril - 15-2015. Aceptado para publicación: septiembre - 26- 2015.

\section{RESUMEN}

Introducción: la enfermedad de Menetrier es poco frecuente en pediatría, sin etiología establecida, se le ha asociado a infecciones en su mayoría virales y bacterianas capaces de generar un proceso inflamatorio que desencadena pérdida de proteínas con clínica de dolor abdominal, náuseas, vómitos y edemas. Con resolución espontánea luego de 4-6 semanas sin secuelas. El objetivo es describir un caso de enfermedad de Menetrier en pediatría.

Caso clínico: preescolar masculino de tres años edad, sin antecedentes perinatales, con cuadro de cinco días de vómitos, diarrea abundante, no disentérica y fiebre; en los últimos dos días con edema bipalpebral. Luego del examen coprológico presentó: quistes de Giardia lamblia, manejado con metronidazol. Es remitido al Hospital Infantil Napoleón Franco Pareja en Cartagena-Colombia por evidencia de edema bipalpebral. En valoración de urgencias se encontró niño en buenas condiciones generales, con facie de enfermedad aguda, con signos vitales estables, hemograma con ligera leucocitosis, extendido de sangre periférica con granulaciones tóxicas en los neutrófilos, parcial de orina sin proteinuria, albúmina sérica $1.26 \mathrm{gr} / \mathrm{dl}$, transaminasas normal, ionograma normal, perfil lipídico normal, ecografía abdominal con moderada distensión de asas intestinales, endoscopia digestiva alta con hipertrofia de pliegues en mucosa de cuerpo gástrico, hiperemia y friabilidad sin erosiones. La biopsia mostró gastropatía crónica activa con marcada hiperplasia foveolar, compatible con enfermedad de Menetrier. Durante su estancia se administró albúmina $1 \mathrm{gr} / \mathrm{kg}$ por cuatro días con resolución del edema y proteinograma, se da egreso con niveles de albúmina normales y en sus controles posteriores, el niño continuó asintomático. Con resolución completa de su cuadro clínico.

1 Médico. Especialista en Pediatría. Grupo de investigación en Gastroenterología Pediátrica. Centro de Investigación y Docencia. Hospital Infantil Napoleón Franco Pareja. Cartagena. Colombia.

2 Médico. Especialista en Pediatría. Docente Departamento de Pediatría. Facultad de Medicina. Universidad de Cartagena. Colombia.

3 Médico. Especialista en Patología. Docente Departamento Diagnóstico. Facultad de Medicina. Universidad de Cartagena. Colombia.

4 Médico. Director del Grupo de Investigación en Economía en Salud. Universidad de Cartagena. Colombia.

5 Médico. Estudiante de Postgrado. Pediatría. Facultad de Medicina. Universidad de Cartagena. Cartagena. Colombia. 
ISSN: 2215-7840, 6(2), julio-diciembre 2015, De Vivero-Camacho Rodrigo, Muñoz-Álvarez Nelson, Redondo-Bermúdez Cesar, Alvis-Guzmán Nelson, Tovar-Correa Luz Esthella

Conclusión: la enfermedad de Menetrier en pediatría se autolimita con una duración de 4 - 6 semanas puede cursar con manifestaciones de dolor abdominal, vómitos, náuseas y edemas. Con las manifestaciones clínicas y hallazgo endoscópico de hipertrofia de pliegues de fondo y cuerpo gástrico se puede presumir el diagnóstico. Se confirma con reporte histopatológico, el curso natural de esta enfermedad es benigno, con resolución completa de los síntomas. Rev.cienc.biomed. 2015; 6(2):354-359

PALABRAS CLAVE

Enfermedad de Menetrier; Hipoalbuminemia; Edema; Citomegalovirus.

\section{SUMMARY}

Introduction: Menetrier's disease is rare in pediatrics. With no established etiology has been associated with viral and bacterial infections most of them able to generate an inflammatory process and protein-loosing with clinical abdominal pain, nausea, vomiting and edema, spontaneous resolution after 4-6 weeks without sequelae. The main objective is to describe a menetrier's disease case in a male child in pediatric.

Clinical case: a three years old male child without perinatal history, with clinical profile of five days with vomiting, abundant diarrhea, not dysentery, and fever, in the last two days with bipalpebral edema. After the cropology test the patient presented Giardia lamblia cysts, treated with metronidazole. The child is remitted to Hospital Infantil Napoleón Franco Pareja in Cartagena-Colombia, because of evidence of edema bipalpebral. In the emergency room male child presented good general condition with acute illness, stable vital signs, blood count with mild leukocytosis, blood smear with toxic granulations in neutrophils, partial urine without proteinuria, serum albumin $1.26 \mathrm{~g} . . / \mathrm{dl}$. normal transaminases, normal serum electrolytes, normal lipid profile, ultrasound with distended bowel moderate, upper endoscopy with hypertrophy folds mucosa and gastric body, hyperemia and friability without erosions. The biopsy showed chronic active gastropathy with marked foveal hyperplasia compatible with Menetrier disease. During his hospitalization was administered albumin $1 \mathrm{~g} / \mathrm{kg}$ for four days with resolution of edema and serum protein, the patient was discharged with normal albumin and subsequent controls and then he continued asymptomatic with complete resolution of the clinical disease.

Conclusion: Menetrier's disease in children is self-limiting with duration from 4 to 6 weeks; it can present abdominal pain, vomiting, nausea and edema. With the clinical and endoscopic findings of gastric folds hypertrophy can be presumed diagnosis. It is confirmed by histopathological report; the natural course of this disease is benign, with complete resolution of symptoms. Rev.cienc.biomed. 2015; 6(2):354-359

\section{KEYWORDS}

Menetrier's disease; Hypoalbuminemia; Edema; Cytomegalovirus.

\section{INTRODUCCIÓN}

La enfermedad de Menetrier es una rara enteropatía en la edad pediátrica, caracterizada por hipertrofia gástrica, con especial predominio en el fondo y cuerpo, asociada a pérdida patológica de proteínas (1-3). La entidad se ha encontrado asociada en la edad pediátrica a infección por citomegalovirus $(4,5)$.

Los pacientes pueden presentar dolor abdominal $(65 \%)$, anorexia (45\%), náuseas o vómitos $(38 \%)$, pérdida de peso $(45 \%)$ y edema $(38 \%)$. Es frecuente la hipoalbuminemia inferior a $3.5 \mathrm{~g} / \mathrm{L}(81 \%)$ y anemia, debido a la pérdida de sangre a nivel gástrico y/o hipoclorhidria con disminución de la absorción de hierro y vitamina B-12 (6-8).
El trastorno fue descrito por primera vez en 1888 por el patólogo francés Pierre Eugene Menetrier (1859-1935), quien observó cambios hiperplásicos en la mucosa gástrica de algunos cadáveres (9).

En el presente reporte se describe un caso de enfermedad de Menetrier asociada a infección por citomegalovirus en un paciente pediátrico.

\section{CASO CLÍNICO}

Paciente masculino de tres años de edad, quien ingresó en noviembre del 2012 al servicio de urgencias del Hospital Infantil Napoleón Franco Pareja de Cartagena-Colombia, remitido de área rural, municipio de San 
Juan Nepomuceno-Bolívar, por un cuadro clínico de cinco días de evolución. Presentaba vómitos de contenido alimentario, deposiciones diarreicas líquidas abundantes, no disentéricas, fiebre en los últimos dos días y edema bipalpebral.

Al ingreso la madre negó disuria, tenesmo o coluria. Consultó inicialmente al Centro de Salud de San Juan Nepomuceno donde realizaron coprológico y encontraron quistes de Giardia lamblia, para lo cual recibió tratamiento con Metronidazol.

Como antecedentes perinatales: niño producto del segundo embarazo a término, nacido por parto vaginal sin complicaciones, pesó al nacer 3.500 gramos y recibió lactancia materna exclusiva los primeros cuatro meses de vida. Recibió desde entonces Nestogeno-1 con leche materna, a partir de los seis meses se inició alimentación complementaria con papillas y sopas de pollo, desde el primer año de edad su alimentación fue igual a la de los adultos: con cinco comidas al día, tres de ellas principales y dos meriendas. Tenía esquema de vacunación completo según el PAI y no presentaba antecedentes de patologías. Su desarrollo psicomotor fue alcanzado normalmente sin ningún indicador de retraso en el mismo.

Se encontró paciente en buen estado músculo nutricional, con facies de enfermedad aguda, hidratado y edema palpebral bilateral. Peso: $13 \mathrm{~kg}$, talla: $96 \mathrm{~cm}$, temperatura: $37.5^{\circ} \mathrm{C}$, frecuencia respiratoria: 25 por minuto, frecuencia cardiaca: 128 x min, Glasgow 15/15. Leve palidez mucocutánea, cuello simétrico sin adenopatías, tórax simétrico sin signos de dificultad respiratoria, ruidos cardiacos rítmicos sin soplos, abdomen con distención, sin masas, megalias ni signos de irritación peritoneal y con onda ascítica negativa. Se realizó impresión clínica de parasitosis intestinal y se decidió dejar en observación para vigilancia de tolerancia a la vía oral y estudio de su edema.

En estudios de laboratorio se encontró, hemoglobina: $13.2 \mathrm{~g} / \mathrm{dl}$, hematocrito: $39.7 \%$, VCM: 75fl, CHCM: $33.3 \mathrm{gr} / \mathrm{dl}$, leucocitos: 15 $100 \mathrm{ml} / \mathrm{mm} 3$, neutrófilos: 67\%, linfocitos: 33\%, plaquetas: 42 0000/mm2. Extendido de sangre periférica sin alteraciones en la serie roja, serie blanca aumentada en número y morfología, con algunas granulaciones tóxicas en PMN. El parcial de orina reportó color amarillo, ph: 5.0, densidad: 1015, bacterias escasas, células: 1-3xcampo, proteínas negativas, acetona: $15 \mathrm{mg} / \mathrm{dl}$, albumina sérica: $1.26 \mathrm{gr} / \mathrm{dl}$, globulina: $1.39 \mathrm{gr} / \mathrm{dl}$, relación A/G: 0.91, BUN: $24.6 \mathrm{mg} / \mathrm{dl}$, creatinina: $0.73 \mathrm{mg} / \mathrm{dl}$, urea: $52.6 \mathrm{mg} / \mathrm{dl}$, SGOT: 49 UI/L, SGPT: $38 \mathrm{UI} / \mathrm{L}$, sodio: $134.5 \mathrm{mEq} / \mathrm{L}$, fósforo: $4.59 \mathrm{mg} / \mathrm{dl}$, magnesio: $4.2 \mathrm{mg} / \mathrm{dL}$., potasio: $4.0 \mathrm{mEq} / \mathrm{L}$, proteinuria en 24 horas: $42.75 \mathrm{mg} / 24$ horas, colesterol: $97 \mathrm{mg} /$ dl, LDL-colesterol: 47.9 mg/dL, HDL-colesterol: $16 \mathrm{mg} / \mathrm{dL}$ y triglicéridos: $165.3 \mathrm{mg} / \mathrm{dL}$. Serología para leptospira IgM e IgG: negativas. Test Sudan-III en materia fecal: Negativa. Ig M Citomegalovirus: positiva, 1.65 (> 1.0). PCR positivo: 231 copias. Ig G Helicobacter Pylori: negativa. Alfa-1-antitripsina en materia fecal: negativo.

Ecografía de abdomen total: moderada distensión de asas intestinales. Esofagogastroduodenoscopia: esófago y duodeno de apariencia normal, hipertrofia en pliegues de la mucosa de cuerpo gástrico, hiperemia y friabilidad, sin erosiones ni úlceras.

Colonoscopía: mucosa del colon distal de aspecto nodular y sin ulceraciones. En ambos estudios se tomaron muestras para estudio de anatomía patológica.

Prueba de ureasa: negativa.

La biopsia de estómago reportó: mucosa gástrica con marcada hiperplasia foveolar, algunas glándulas con cambios epiteliales reactivos con tendencia a la dilatación quística. En la lámina propia se observó infiltrado inflamatorio crónico mediado por linfocitos y plasmocitos, con marcada actividad sin evidencia de H. Pilory ni metaplasia. Se consideró proceso inflamatorio, gastropatía crónica activa con marcada hiperplasia foveolar. Figura $\mathrm{N}^{0} 3$ y No4. Duodeno y esófago, sin alteraciones. Biopsia de colon reportó: infiltrado inflamatorio crónico severo, presencia de neutrófilos a nivel de la lámina propia, sin evidencia de micro abscesos crípticos, pérdida del moco glandular ni presencia de granulomas. 

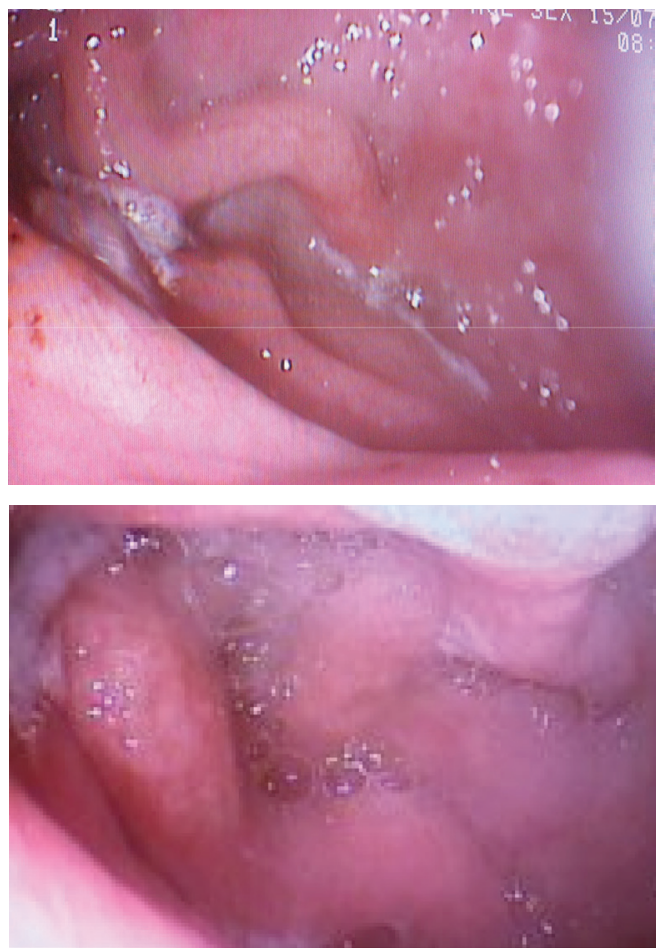

Figura $\mathbf{N}^{\circ} \mathbf{1}$ y N02. Engrosamiento de la mucosa gástrica con marcada inflamación.

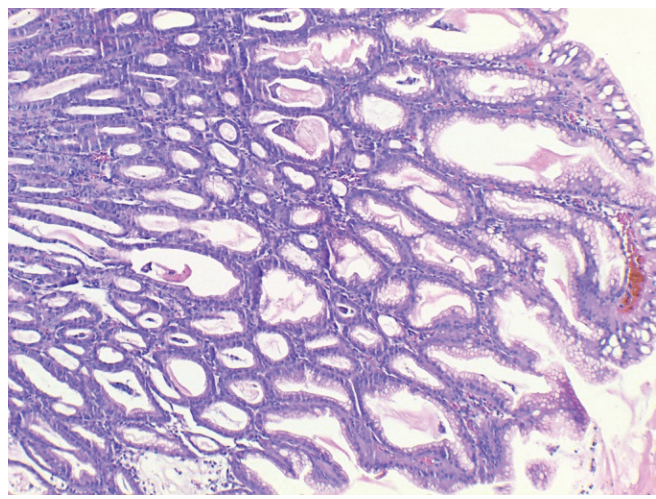

Figura N03. Mucosa gástrica. Quistes que por poco penetran la capa muscular de la mucosa extendiéndose a la submucosa. Hematoxilina \& Eosina. $40 x$



Figura N04. Intensa proliferación de células superficiales que dan a las glándulas del fondo, aspecto de sacacorchos. Hematoxilina \& Eosina. 40 x
Durante la hospitalización se administró albúmina a $1 \mathrm{gr} / \mathrm{kg}$ por cuatro días. El paciente evolucionó adecuadamente, con disminución del edema, resolución del cuadro diarreico, adecuada tolerancia a la vía oral, llegándose a niveles de albúmina en $3.0 \mathrm{~g} / \mathrm{dl}$. Fue egresado con recomendaciones alimentarias, a las cuatro semanas fue evaluado, encontrándose asintomático, control de albúmina de $3.7 \mathrm{~g} / \mathrm{dl}$, siendo dado de alta con la recomendación de controles pediátricos rutinarios. En el control posterior, asintomático. Se le indicó continuar con su pediatra y se dio egreso por el servicio de gastroenterología pediátrica.

\section{DISCUSIÓN}

Este es el segundo caso reportado en la ciudad de Cartagena en los últimos 15 años en pacientes pediátricos (9). Constituye una enfermedad rara con una incidencia $<1$ por 200.000, con una distribución bimodal afectando niños a menudo menores de 10 años y adultos de 30-60 años con un pico a los 55 años. Con preponderancia masculina tanto en jóvenes como en adultos (10).

Los casos encontrados se han asociado a infección por citomegalovirus y a $\mathrm{H}$. Pylori, aproximadamente $1 / 3$ de los casos en pediatría se encuentra coinfección por citomegalovirus y evidencia endoscópica de la enfermedad, como la descrita en nuestro caso (11).

De acuerdo a la fisiopatología, la enfermedad de Menetrier se evidencia por los cambios histológicos que incluyen la hiperplasia foveolar (ampliación de las células de la superficie de las mucosas), dilatación quística y reducción del número de células parietales y principales $(12,13)$. Esto conlleva a inadecuada producción de ácido clorhídrico y factor intrínseco (14), generando producción excesiva de moco, malabsorción y exudación de proteínas que son responsables de edemas e hipoalbuminemia tal como se presentó en el caso (15).

Se ha encontrado también enfermedad de Menetrier asociada a infección por mycoplasma pneumoniae y $\mathrm{H}$. Pylori (16). Sin embargo, la relación más frecuente en pediatría, es con infección por citomegalovirus $(4,5)$ 
el cual es un virus herpes tipo 5 que puede infectar por primoinfección, reactivación o reinfección. Es muy difícil establecer si la infección por citomegalovirus es la causante de la enteropatía perdedora de proteínas. La serología tipo IgM puede mantenerse positiva durante largo tiempo y no ser la etiología directa de la enfermedad de Menetrier. En el paciente se encontró IgM positiva y PCR para citomegalovirus, lo cual sumado a los hallazgos de la biopsia que reveló gastropatía hipertrófica, permitieron realizar el diagnóstico.

Por otro lado, se han identificado nuevos métodos diagnósticos de infección por citomegalovirus como inmunohistoquímica de antígeno capsular (17). Mientras se realiza la endoscopia que permita visualizar la hipertrofia de la mucosa gástrica -esta es la prueba estándar de oro para el diagnósticocon hallazgos de cambios morfológicos de predominio en fondo y en el cuerpo gástrico, áreas a las cuales se tomará biopsia y serán sometidas a tinciones, mediante las cuales se pueden identificar las inclusiones citomegálicas del virus herpes 5 .

Existe un examen con mejor sensibilidad: la PCR (reacción en cadena de la polimerasa) para citomegalovirus, el cual brinda un diagnóstico más exacto de la infección (18). En este caso, la muestra debe ser de la biopsia tomada de la mucosa gástrica, con el inconveniente que no todas las instituciones de salud cuentan con este servicio debido al alto costo.
El manejo de la enfermedad de Menetrier consiste en brindar el soporte adecuado, ya que es una enfermedad autolimitada con duración de 1 a 2 meses $(19,20)$. Se recomienda infusión de albúmina a 1 $\mathrm{gr} / \mathrm{kg} /$ día y dieta hiperproteica (17). En niños no se recomienda la terapia antiviral (2). Esta solo se recomienda en el manejo de pacientes que tienen infección por VIH con recuento de CD4 $<50$, sin tener en cuenta la carga viral, y en recién nacidos con hallazgos de enfermedad congénita por citomegalovirus (21).

En el caso descrito, el niño evolucionó de manera satisfactoria, se hizo reposición de albúmina y se dio egreso con niveles séricos de albúmina normales, en sus controles posteriores a los dos semanas y al mes, continuó asintomático con proteinograma normal. Por lo cual se dio egreso con gastroenterología pediátrica y se indicó continuar sus controles con pediatría.

\section{CONCLUSIÓN}

Se debe sospechar siempre enfermedad de Menetrier ante todo en pacientes con síntomas gastrointestinales, con hipoalbuminemia de causa no renal.

CONFLICTOS DE INTERESES: ninguno que declarar.

FINANCIACIÓN: recursos propios para los autores.

\section{REFERENCIAS BIBLIOGRÁFICAS}

1. Cieslak TJ, Mullett CT, Puntel RA, Latimer JS. Menetrier's disease associated with cytomegalovirus infection in children: report of two cases and review of the literature. The Pediatric infectious disease journal. 1993;12(4):340-3.

2. Rich A, Toro TZ, Tanksley J, Fiske WH, Lind CD, Ayers GD, et al. Distinguishing Menetrier's disease from its mimics. Gut. 2010;59(12):1617-24.

3. Son KH, Kwak JJ, Park JO. A case of cytomegalovirus-negative Menetrier's disease with eosinophilia in a child. Korean journal of pediatrics. 2012;55(8):293-6.

4. Kovacs AA, Churchill MA, Wood D, Mascola L, Zaia JA. Molecular and epidemiologic evaluations of a cluster of cases of Menetrier's disease associated with cytomegalovirus. The Pediatric infectious disease journal. 1993;12(12):1011-4.

5. Leonidas JC, Beatty EC, Wenner HA. Menetrier disease and cytomegalovirus infection in childhood. Am J Dis Child. 1973;126(6):806-8.

6. Konstantinidou AE, Morphopoulos G, Korkolopoulou P, et al. Menetrier disease of early infancy: a separate entity? . Journal of Pediatric Gastroenterology and Nutrition. 2004;39:17782.

7. Dempsey PJ, Goldenring JR, Soroka CJ, Modlin IM, McClure RW, Lind CD, et al. Possible role of transforming growth factor alpha in the pathogenesis of Menetrier's disease: supportive evidence form humans and transgenic mice. Gastroenterology. 1992;103(6):1950-63.

8. Meuwissen SG, Ridwan BU, Hasper HJ, Innemee G. Hypertrophic protein-losing gastro- 
ISSN: 2215-7840, 6(2), julio-diciembre 2015, De Vivero-Camacho Rodrigo, Muñoz-Álvarez Nelson, Redondo-Bermúdez Cesar, Alvis-Guzmán Nelson, Tovar-Correa Luz Esthella

pathy. A retrospective analysis of 40 cases in The Netherlands. The Dutch Menetrier Study Group. Scand J Gastroenterol Suppl. 1992;194:1-7.

9. De Vivero-Camacho R. Enfermedad de menetriere en niños, reporte de un caso. Pediatría [Internet]. 2002; 37(1).

10. Fishman EK, Urban BA, Hruban RH. CT of the stomach: spectrum of disease. Radiographics. 1996;16 (5): 1035-54.

11. Tokuhara D, Okano Y, Asou K, Tamamori A, Yamano T. Cytomegalovirus and Helicobacter pylori co-infection in a child with Menetrier disease. European journal of pediatrics. 2007; 166(1):63-5.

12. Occena RO, Taylor SF, Robinson CC, Sokol RJ. Association of cytomegalovirus with Menetrier's disease in childhood: report of two new cases with a review of literature. Journal of pediatric gastroenterology and nutrition. 1993;17(2):217-24.

13. Okanobu H, Hata J, Haruma K, Hara M, Nakamura K, Tanaka S, et al. Giant gastric folds: differential diagnosis at US. Radiology. 2003;226(3):686-90.

14. Ba-Ssalamah A, Prokop M, Uffmann M, Pokieser P, Teleky B, Lechner G. Dedicated multidetector CT of the stomach: spectrum of diseases. Radiographics : a review publication of the Radiological Society of North America, Inc. 2003;23(3):625-44.

15. Cotran RS, Kumar V, Collins T. Robbins Pathologic basis of disease. 6th ed. Philadelphia, Pa: WB Saunders; 1999.

16. Coffey RJ, Washington MK, Corless CL, Heinrich MC. Menetrier disease and gastrointestinal stromal tumors: hyperproliferative disorders of the stomach. The Journal of clinical investigation. 2007;117(1):70-80.

17. Ben Amitai D, Zahavi I, Dinari G, Garty BZ. Transient protein-losing hypertrophic gastropathy associated with Mycoplasma pneumoniae infection in childhood. Journal of pediatric gastroenterology and nutrition. 1992;14(2):237-9.

18. Cheong JL, Cowan FM, Modi N. Gastrointestinal manifestations of postnatal cytomegalovirus infection in infants admitted to a neonatal intensive care unit over a five year period. Archives of disease in childhood Fetal and neonatal edition. 2004;89(4):F367-9.

19. Boeckh M, Huang M, Ferrenberg J, Stevens-Ayers T, Stensland L, Nichols WG, et al. Optimization of quantitative detection of cytomegalovirus DNA in plasma by real-time PCR. Journal of clinical microbiology. 2004;42(3):1142-8.

20. Hoffer V, Finkelstein Y, Balter J, Feinmesser M, Garty BZ. Ganciclovir treatment in Menetrier's disease. Acta Paediatr. 2003;92(8):983-5.

21. Iwanaga M, Zaitsu M, Ishii E, Nishimura $Y$, Inada $S$, Yoshiki H, et al. Protein-losing gastroenteropathy and retinitis associated with cytomegalovirus infection in an immunocompetent infant: a case report. European journal of pediatrics. 2004;163(2):81-4.

22. Demmler GJ. Infectious Diseases Society of America and Centers for Disease Control. Summary of a workshop on surveillance for congenital cytomegalovirus disease. Reviews of Infectious Diseases. 1991;13(2):315-29.

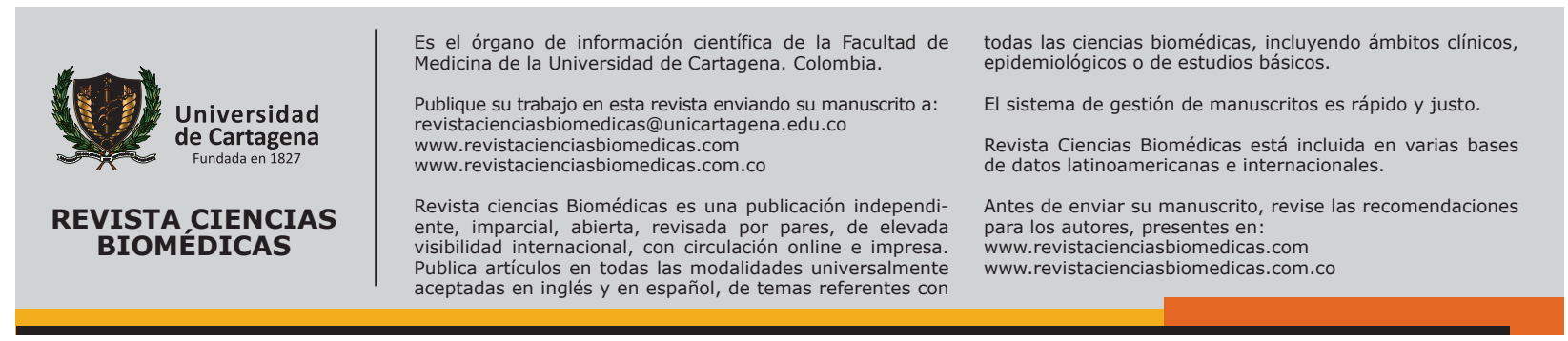

